McGill/92-22

hep-ph/9207207

rev. August 1992

\title{
Majorons without Majorana Masses and Neutrinoless Double Beta Decay
}

\author{
C.P. Burgess and J.M. Cline* \\ McGill University \\ 3600 University Street \\ Montréal, Québec, Canada H3A 2T8
}

\begin{abstract}
We explain excess events near the endpoints of the double beta decay $(\beta \beta)$ spectra of several elements, using the neutrinoless emission of massless Goldstone bosons. Models with scalars carrying lepton number -2 are proposed for this purpose so that ordinary neutrinoless $\beta \beta$ is forbidden, and we can raise the scale of global symmetry breaking above the $10 \mathrm{keV}$ scale needed for observable emission of conventional Majorons in $\beta \beta$. The electron spectrum has a different shape, and the rate depends on different nuclear matrix elements, than for the emission of ordinary Majorons.
\end{abstract}

* Present address: Theoretical Physics Institute, University of Minnesota, Minneapolis, MN 55455, USA 
One of the deep questions of particle physics is whether there exist any fundamental particles of spin zero. A candidate is the Majoron, the massless Goldstone boson that would exist if lepton number $(L)$ were spontaneously broken [1]. Majorons would be hard to detect since they couple directly only to neutrinos, with a strength proportional to the neutrino masses divided by the $L$-breaking scale $v$ :

$$
g_{\nu}=m_{\nu} / v
$$

If $g_{\nu}$ were large enough, Majorons could be seen in double beta decay [2] [3], a rare process now observed in seven elements. Besides the usual neutrino-emitting mode $\beta \beta_{2 \nu}$, a Majoron could be emitted through annihilation of the virtual neutrinos, $\beta \beta_{M}$. The $\beta \beta_{M}$ and $\beta \beta_{2 \nu}$ signals are distinguishable since for $\beta \beta_{M}$ the decay energy is shared among fewer particles, skewing its electron spectrum toward higher energies.

In fact, a mysterious excess of high-energy electrons is seen in the $\beta \beta$ spectra for several elements. Such an observation was first made in 1987 for ${ }^{76} \mathrm{Ge} \rightarrow{ }^{76} \mathrm{Se}+2 e^{-}$by Avignone et al. [4], although they, and other groups, subsequently excluded a signal at the original level [5]. Now the UC Irvine group also finds excess numbers of electrons near but below the endpoints for ${ }^{100} \mathrm{Mo},{ }^{82} \mathrm{Se}$ and ${ }^{150} \mathrm{Nd}$, with a statistical significance of $5 \sigma$ [6]. Such events also persist in ${ }^{76} \mathrm{Ge}$ [7], at approximately a tenth of the original rate.

Formerly all these $\beta \beta$ measurements were compared with the Gelmini-Roncadelli (GR) model [8] [3], which however has been ruled out by LEP's bounds on the invisible Z width. In this model lepton number was spontaneously broken by an electroweak-triplet Higgs field, resulting in both a Majorana mass and a direct coupling to the Majoron for $\nu_{e}$, as in eq. (11). The presently observed excess events could then be explained by $\beta \beta_{M}$ if $g_{\nu_{e}} \sim 1 \times 10^{-4}$, and the absence of $\beta \beta_{0 \nu}$ decay — which would appear as a line at the endpoint in the sum-energy spectrum - requires $m_{\nu_{e}} \lesssim 1 \mathrm{eV}$. Eq. (1) then implies that the triplet VEV must be unnaturally small,

$$
v<10 \mathrm{keV} \text {. }
$$

(A similar bound follows from astrophysical considerations.)

With the demise of the GR model, it appeared that there existed no models capable of predicting $\beta \beta_{M}$ at an observable rate. In this Letter we propose an alternative broad class of Majoron models which might explain the excess events, while still preserving the 
agreement between theory and geochemical experiments for the $\beta \beta$ decay rate of ${ }^{128} \mathrm{Te}$, ${ }^{130}$ Te and ${ }^{238} \mathrm{U}$ [9].

We start by describing some generic features of $\beta \beta_{M}$ decays, and then turn to modelspecific issues. First, agreement with the $Z$-width measurement rules out a direct Majoron coupling to the $Z$ boson. Lepton number breaking, if it occurs, must come from an electroweak-singlet field. Majorons therefore cannot have renormalizable couplings to $\nu_{e}$; instead, $\beta \beta_{M}$ proceeds through mixing of $\nu_{e}$ with sterile neutrinos that couple to Majorons.

Next one must explain how $\beta \beta_{M}$ but not $\beta \beta_{0 \nu}$ events are seen, since lepton-breaking generically gives both effects. The GR model did so by requiring a small lepton-number breaking scale as in eq. (2), and the same expedient is used by alternate models where electroweak-singlets rather than triplets do the lepton-breaking. But once such a small scale is introduced by hand it is unnecessary for the scalar emitted in $\beta \beta_{M}$ to be a Goldstone boson; it could just as well be given a mass of $10 \mathrm{keV}$. We refer to these as 'ordinary Majorons' (and denote their emission by $\beta \beta_{O M}$ ) since they share many features (electron spectra, etc.) of the GR model. These models all suffer from a hierarchy problem since the lepton-breaking VEV is not stable under renormalization and so must be artificially fine-tuned [10].

Here we introduce a class of models that do not require such a small scale, inspired by realizing that the smallness of $v$ in eq. (2) comes from the bound on $\beta \beta_{0 \nu}$ decay. This restriction disappears if a lepton number, $L$, carried by electrons, remains unbroken, so that $\beta \beta_{0 \nu}$ is completely forbidden. Then $\beta \beta_{M}$ occurs only if the scalar particle itself is 'charged,' i.e., it carries $L(\varphi)=-2$, in units where $L\left(e^{-}\right)=+1$. Fine-tuning of the scalar mass is avoided if the scalar is a Goldstone boson, a 'charged Majoron.' All of this can be realized in a simple way, using for example a global $S U(2)_{S} \times U(1)_{L^{\prime}}$ symmetry broken down to $U(1)_{L}$. (The $U(1)_{L^{\prime}}$ factor exists so that Majoron couplings to ordinary charged leptons can be avoided.) Two of the resulting Goldstone bosons then carry the unbroken charge, just as the would-be Goldstone bosons that make up the longitudinal $W^{ \pm}$'s carry electric charge in the Standard Model. We call all such models 'charged' Majoron models and denote the resulting process $\beta \beta_{c M}$.

In what follows we compare charged and ordinary Majoron models with each another and the data. We will: $(i)$ show how the models can be experimentally distinguished by the predicted shape of the sum electron spectrum, and ( $i i)$ find the necessary conditions for getting a large enough rate of $\beta \beta_{M}$. 
i. The electron spectrum. The decay rates for $\beta \beta_{2 \nu}, \beta \beta_{O M}$ and $\beta \beta_{c M}$ all have the form

$$
d \Gamma(\beta \beta)=\frac{G_{F}^{4}}{4 \pi^{3}}|\mathcal{A}(\beta \beta)|^{2} d \Omega_{n},
$$

where $G_{F}$ is the Fermi constant, $\mathcal{A}$ is a matrix element (more about which later), and $d \Omega_{n}$ is the phase space, a function of the momenta and energies of the two outgoing electrons,

$$
d \Omega_{n}=\frac{1}{64 \pi^{2}}\left(Q-\epsilon_{1}-\epsilon_{2}\right)^{n} \prod_{k=1}^{2} p_{k} \epsilon_{k} F\left(\epsilon_{k}\right) d \epsilon_{k} .
$$

$F(\epsilon)$ is the Fermi function, equal to 1 when the nuclear charge vanishes, and $Q \simeq 1-$ $2 \mathrm{MeV}$ is the endpoint energy for the final-state electrons.

The integer $n$ in eq. (44) depends on the decay channel: $n=5$ for $\beta \beta_{2 \nu}, 3$ for $\beta \beta_{c M}$, and 1 for $\beta \beta_{\text {oM }} . n$ controls the shape of the electron energy spectrum, because for these decays all lepton energies are $\ll p_{F} \sim 100 \mathrm{MeV}$, the nuclear scale that determines the matrix element $\mathcal{A}$. Thus it is a good approximation - relative error of order $Q / p_{F} \sim 1 \%$ - to neglect $\mathcal{A}$ 's dependence on the outgoing lepton energies. In particular, near the endpoint the electron spectrum in $\beta \beta_{c M}$ goes like $(Q-E)^{3}$ as $E$, the sum of electron energies, approaches $Q$. This is intermediate between $(Q-E)$ for $\beta \beta_{O M}$, and $(Q-E)^{5}$ for $\beta \beta_{2 \nu}$. The spectra are plotted in Fig. 1. The distinction between $\beta \beta_{C M}$ and $\beta \beta_{O M}$ is one of our main results.

Figure 1. The electron sum-energy spectrum for $\beta \beta_{2 \nu}$ (dashed), $\beta \beta_{c M}$ (solid) and $\beta \beta_{O M}$ (dot-dashed) decays. 
We emphasize that the spectral shape difference characterizes these two classes of models, independently of any model-specific details. This traces to the vanishing of $\beta \beta_{c M}$ amplitudes at zero Majoron momentum, $q=0$, due to the fact that Goldstone bosons are always derivatively coupled. The puzzle is why $\beta \beta_{O M}$ amplitudes do not also vanish at $q=0$. There one finds that graphs with Majoron Brehmsstrahlung off the electron lines survive as $q \rightarrow 0$ because the internal electron propagator diverges in this limit, leaving a finite result. The same cannot happen for $\beta \beta_{c M}$ because the electron-Majoron coupling is forbidden by the unbroken lepton symmetry in these models.

ii. The decay rate. Computing the absolute rate requires knowing the matrix element, $\mathcal{A}$, of eq. (3). Consider arbitrary Yukawa couplings between a set of neutrinos and a complex scalar field, $\varphi$,

$$
\mathcal{L}_{M}=-\frac{1}{2} \bar{\nu}_{i}\left(a_{i j} P_{L}+b_{i j} P_{R}\right) \nu_{j} \varphi^{*}+c . c .
$$

Goldstone boson couplings are included here as a special case: if $f$ is the decay constant, $X_{i j}$ the conserved charge and $m_{i j}$ the left-handed neutrino mass matrix, then $a_{i j}$ takes the form $a=-i\left(X^{T} m+m X\right) / f$, and similarly for $b_{i j}$. Real scalar fields are also easily incorporated by taking $b=a^{*}$ in all results. For example the GR Majoron coupling arising from a triplet interaction $\mathcal{L}=\frac{1}{2} g \bar{L}_{e} P_{L} \tau_{a} L_{e} \Delta^{a}+$ c.c. would give $b_{e e}^{*}=a_{e e}=i g$.

To lowest (zeroeth) order in the scalar energy we get eq. (3) with $n=1$ and

$$
\mathcal{A}\left(\beta \beta_{O M}\right)=\sum_{i j} V_{e i} V_{e j} \int \frac{d^{4} p}{(2 \pi)^{4}} \frac{\left(4 w_{1}-w_{2}+p^{2} w_{5}\right)\left(a_{i j} m_{\nu_{i}} m_{\nu_{j}}-p^{2} b_{i j}\right)}{\left(p^{2}+m_{\nu_{i}}^{2}-i \varepsilon\right)\left(p^{2}+m_{\nu_{j}}^{2}-i \varepsilon\right)} .
$$

$V_{e i}$ is the mixing matrix element for finding the mass eigenstate $\nu_{i}$ in an electron chargedcurrent weak interaction. The form factors $w_{a}$ parametrize the matrix element of the hadronic weak currents between initial and final nuclei $N$ and $N^{\prime}$,

$$
\begin{aligned}
W_{\alpha \beta} \equiv & \int d^{4} x\left\langle N^{\prime}\left|T^{*}\left[J_{\alpha}(x) J_{\beta}(0)\right]\right| N\right\rangle e^{i p x} \\
= & w_{1} \eta_{\alpha \beta}+w_{2} u_{\alpha} u_{\beta}+w_{3}\left(p_{\alpha} u_{\beta}-p_{\beta} u_{\alpha}\right)+ \\
& \quad+w_{4} \epsilon_{\alpha \beta \sigma \rho} u^{\sigma} p^{\rho}+w_{5} p_{\alpha} p_{\beta},
\end{aligned}
$$

and are functions of the invariants $p^{2}$ and $u \cdot p . u_{\alpha}$ is the four-velocity of the initial and final nuclei, which are equal since we neglect all dependence in $W_{\alpha \beta}$ on the final-state momenta. 
Although eq. (6) suffices for $\beta \beta_{O M}$ it happens that for $\beta \beta_{C M}$ this lowest-order result vanishes, making it necessary to work to next-higher order in the outgoing Majoron momentum, and leading to the difference in electron spectra (above). The result has the form of eq. (3), but with $n=3$ and $\left|\mathcal{A}\left(\beta \beta_{c M}\right)\right|^{2}=\left|\mathcal{U}\left(\beta \beta_{c M}\right)\right|^{2}+\left|\mathcal{V}\left(\beta \beta_{c M}\right)\right|^{2}$, where

$$
\begin{aligned}
& \mathcal{U}\left(\beta \beta_{c M}\right)=\sum_{i j} V_{e i} V_{e j} b_{i j} \int \frac{d^{4} p}{(2 \pi)^{4}} \frac{2 \vec{p}^{2}\left(w_{3}-2 i w_{4}\right)}{\left(p^{2}+m_{\nu_{i}}^{2}-i \varepsilon\right)\left(p^{2}+m_{\nu_{j}}^{2}-i \varepsilon\right)} \\
& \mathcal{V}\left(\beta \beta_{c M}\right)=\sum_{i j} V_{e i} V_{e j} b_{i j} \int \frac{d^{4} p}{(2 \pi)^{4}} \frac{p^{0}\left(w_{2}-p^{2} w_{5}\right)}{\left(p^{2}+m_{\nu_{i}}^{2}-i \varepsilon\right)\left(p^{2}+m_{\nu_{j}}^{2}-i \varepsilon\right)} .
\end{aligned}
$$

Notice that whereas $\mathcal{A}\left(\beta \beta_{O M}\right)$ has the same combination of form factors as $\beta \beta_{0 \nu}$, $\beta \beta_{c M}$ depends on different nuclear matrix elements. (For $\beta \beta_{0 \nu}, \mathcal{A} \propto \sum_{i} V_{e i}^{2} m_{\nu_{i}}$ $\int\left[d^{4} p /(2 \pi)^{4}\right]\left[\left(4 w_{1}-w_{2}+p^{2} w_{5}\right) /\left(p^{2}+m_{\nu_{i}}^{2}-i \varepsilon\right)\right]$; these are related to the Fermi- and Gamow-Teller-type form factors by $w_{1}=\frac{1}{3} w_{G T}$ and $w_{2}=w_{F}+\frac{1}{3} w_{G T}$.)

A striking feature of both eqs. (6) and (8) is that they vanish if all neutrinos share a common mass, for in this limit the sum over states gives $\sum_{i j} V_{e i} V_{e j} a_{i j}=a_{e e}$. The latter is zero since the $Z^{0}$ width constraint implies that the Majoron cannot directly couple to $\nu_{e}$. As an important corollary, the amplitudes must also vanish if all of the $\nu_{i}$ are negligibly light compared to the Fermi momentum $p_{F}$, above which the integrals in (66) and (8) are effectively cut off. Thus in any model explaining the excess electron events by Majoron couplings to a virtual neutrino, at least one of the $\nu_{i}$ must have a mass $m_{\nu_{i}} \gtrsim 100 \mathrm{MeV}$.

To make contact with the literature, we use the predictions of the GR model as a benchmark. In this case

$$
\mathcal{A}\left(\beta \beta_{M}\right)_{G R}=\frac{2 \sqrt{2}}{\pi} g_{A}^{2} g_{\mathrm{eff}} \mathcal{M}
$$

$g_{\text {eff }}$ is the GR coupling of $\varphi$ to $\nu_{e}, g_{V}$ and $g_{A}$ are the axial and vector couplings of the nucleon weak currents, and $\mathcal{M}=\left\langle\left[\sigma_{n} \cdot \sigma_{m}-\left(g_{V} / g_{A}\right)^{2}\right] h(r)\right\rangle$ (see eq. (18)). We integrate the phase space $d \Omega_{1}$ above a threshold $E_{\text {th }}$ where the anomalous events begin and the contribution from ordinary $\beta \beta$ decay should be small. Table 1 compares this with the excess events seen by the Irvine group for the elements ${ }^{82} \mathrm{Se},{ }^{100} \mathrm{Mo}$ and ${ }^{150} \mathrm{Nd}[\mathrm{G}$, and in the published spectrum of ${ }^{76} \mathrm{Ge}$ [11]. In all of these cases the excess events comprise $R=2$ to $3 \%$ of the total number observed. We also analyze the geochemically observed decays, 
Table 1: parameters for emission of GR Majorons in double beta decay. $T_{1 / 2}^{-1}$ is the inverse half-life of the anomalous events; note that for the last three elements this is assumed to be the entire rate; $|\mathcal{M}|^{2}$ is the $0 \nu$ matrix element extracted from Staudt et al.; and $R$ is the ratio of anomalous to all events - hypothesized to be 1 for the geochemically observed decays. $\Omega_{1} m_{e}^{7}$ is the total phase space for Majoron events (see eq. (4)), while $E_{\mathrm{th}}(\mathrm{MeV})$ denotes our choice for the threshold value of the sum of the electron energies, above which essentially only excess events appear. $\Delta \Omega_{1} m_{e}^{7}$ is the part of phase space occurring above $E_{\mathrm{th}}$ and $g_{\mathrm{eff}}=g_{e e}$ is the coupling needed to explain the rate using Majoron emission in the GR model.

\begin{tabular}{|c|c|c|c|c|c|c|c|}
\hline Element & $T_{1 / 2}^{-1}\left(\mathrm{y}^{-1}\right)$ & $|\mathcal{M}|^{2} m_{e}^{-2}$ & $R$ & $\Omega_{1}$ & $E_{\text {th }}$ & $\Delta \Omega_{1}$ & $g_{\text {eff }}$ \\
\hline${ }^{76} \mathrm{Ge}$ & $2 \times 10^{-23}$ & $1 \times 10^{5}$ & 0.02 & 2.0 & 1.5 & 0.9 & $1 \times 10^{-4}$ \\
${ }^{82} \mathrm{Se}$ & $2 \times 10^{-22}$ & $9 \times 10^{4}$ & 0.03 & 17 & 2.2 & 7.6 & $8 \times 10^{-5}$ \\
${ }^{100} \mathrm{Mo}$ & $3 \times 10^{-21}$ & $2 \times 10^{4}$ & 0.03 & 34 & 1.9 & 22 & $4 \times 10^{-4}$ \\
${ }^{150} \mathrm{Nd}$ & $3 \times 10^{-20}$ & $1 \times 10^{5}$ & 0.02 & 260 & 2.2 & 155 & $2 \times 10^{-4}$ \\
\hline${ }^{128} \mathrm{Te}$ & $5 \times 10^{-25}$ & $7 \times 10^{4}$ & 1.00 & 0.23 & 0.0 & 0.23 & $4 \times 10^{-5}$ \\
${ }^{130} \mathrm{Te}$ & $1 \times 10^{-21}$ & $5 \times 10^{4}$ & 1.00 & 30 & 0.0 & 30 & $1 \times 10^{-4}$ \\
${ }^{238} \mathrm{U}$ & $5 \times 10^{-22}$ & $3 \times 10^{4}$ & 1.00 & 33 & 0.0 & 33 & $2 \times 10^{-4}$ \\
\hline
\end{tabular}

assuming all the events are due to $\beta \beta_{M}$. Remarkably, $g_{\text {eff }}$ lies in the range $4 \times 10^{-5}$ to $4 \times 10^{-4}$ for all seven elements.

The predictions for Te are of particular interest because of a recent measurement of the ratio of decay rates $\zeta \equiv \Gamma\left({ }^{130} \mathrm{Te}\right) / \Gamma\left({ }^{128} \mathrm{Te}\right)=(2.41 \pm 0.06) \times 10^{3}$ [12]. If the same coupling needed to account for the endpoint anomalies $\left(g_{\text {eff }} \sim 1 \times 10^{-4}\right)$ is used for these decays then the GR and $\beta \beta_{O M}$ models predict too small a ratio: $\zeta\left(\beta \beta_{O M}\right)=(30.4 / 0.23)(5 / 7)=93$. The same is not true for $\beta \beta_{c M}$ models because the rates scale with an extra factor of $Q^{2}$ relative to those for $\beta \beta_{o M}$, and ${ }^{128}$ Te has a small endpoint energy, $Q \approx 0.9 \mathrm{MeV}$. Thus we find $\zeta\left(\beta \beta_{C M}\right)=770$, in much better agreement with the experimental value.

To see whether the ordinary or charged Majoron models can predict a large enough rate, we display the combinations of couplings, defined in (5), that are equivalent to the GR model with coupling $g_{\text {eff }}$, so far as the total rate is concerned. For ordinary Majorons

$$
g_{\mathrm{eff}}=\sum_{i j} V_{e i} V_{e j}\left\langle\frac{1}{p^{2}}\right\rangle^{-1}\left\langle\frac{m_{\nu_{i}} a_{i j} m_{\nu_{j}}-p^{2} b_{i j}}{\left(p^{2}+m_{\nu_{i}}^{2}\right)\left(p^{2}+m_{\nu_{j}}^{2}\right)}\right\rangle .
$$

Here $p$ is the four-momentum of the virtual neutrino, which as indicated by \langle\rangle , is integrated weighted by the nuclear form factors. An estimate for the size of $g_{\mathrm{eff}}$ is therefore obtained 
by replacing $p^{2}$ by the characteristic momentum scale, $p_{F}^{2} \sim(100 \mathrm{MeV})^{2}$. For charged Majorons, in order of magnitude

$$
g_{\mathrm{eff}}=-\sum_{i j} V_{e i} V_{e j}\left\langle\frac{1}{p^{2}}\right\rangle^{-1}\left\langle\frac{Q p b_{i j}}{\left(p^{2}+m_{\nu_{i}}^{2}\right)\left(p^{2}+m_{\nu_{j}}^{2}\right)}\right\rangle X .
$$

The two qualitatively new features here are: (1) the additional suppression by the endpoint energy, $Q / p$, due to the softer electron spectrum of these models, and (2) a ratio, $X$, of the $\beta \beta_{C M}$ to $\beta \beta_{O M}$ nuclear matrix elements. Later we give a more quantitative comparison of these matrix elements, in a specific model.

Consider now a representative model for each class. For $\beta \beta_{O M}$, augment the Standard Model with two Majorana-Dirac sterile neutrinos, $s_{+}$and $s_{-}$, and a complex singlet scalar, $\varphi$. The model consists of interactions that preserve electron-type lepton number, assuming $L_{e}\left(P_{L} s_{ \pm}\right)= \pm 1$, and $L_{e}(\varphi)=-2$. To satisfy the $\beta \beta_{0 \nu}$ constraint we take $\langle\varphi\rangle=0$ and fine-tune the $\varphi$ mass to be $\lesssim 1 \mathrm{MeV}$ as required by the $\beta \beta_{M}$ kinematics. The spectrum contains one Dirac neutrino $\left(\Psi_{R}, \Psi_{L}\right)$ with mass $M, \Psi_{R}=s_{-}^{c}$ and $\Psi_{L}=\nu_{e} \sin \theta+s_{+} \cos \theta$. The massless orthogonal combination $\nu_{e}^{\prime}$ is predominantly $\nu_{e}$. The remaining two neutrinos $\nu_{\mu}$ and $\nu_{\tau}$ acquire Majorana masses at dimension five.

In terms of the relevant Yukawa couplings to $\varphi$,

$$
\mathcal{L}_{y}=-\frac{1}{2} g_{+}\left(\bar{s}_{+} P_{L} s_{+}\right) \varphi-\frac{1}{2} g_{-}\left(\bar{s}_{-} P_{L} s_{-}\right) \varphi^{*}+\text { c.c. }
$$

the effective $\beta \beta_{M}$ coupling in this model becomes

$$
g_{\mathrm{eff}}=\left\langle\frac{1}{p^{2}}\right\rangle^{-1}\left\langle\frac{\left(g_{-} p^{2}-g_{+} M^{2} \cos ^{2} \theta\right) M^{2} \sin ^{2} \theta}{p^{2}\left(p^{2}+M^{2}\right)^{2}}\right\rangle .
$$

Since the light scalars couple to the massless neutrino $\nu_{e}^{\prime}$ through mixing, $\beta \beta_{O M}$ may proceed via the exchange of $\nu_{e}^{\prime}$, with the result $g_{\text {eff }}$ is nonvanishing as $M \rightarrow \infty$ with $\theta$ held fixed. (Decoupling is not violated since $\theta \sim 1 / M$ if dimensionless couplings in the lagrangian are held fixed for $M$ large.) Using $\left\langle p^{2}\right\rangle \sim(100 \mathrm{MeV})^{2}$ and $\theta \sim 0.1$ we find $g_{\text {eff }} \sim 10^{-4}$, as suggested by the $\beta \beta$ anomalies. We choose $M$ above the $K^{ \pm}$meson mass; otherwise a heavy neutrino with such large mixing would have been seen as a peak in the $K \rightarrow e \nu$ [13] or $\pi \rightarrow e \nu$ [14] decay spectra.

For charged Majorons we suppose that the lepton symmetry group surviving at electroweak energies is $G_{L}=S U(2)_{s} \times U(1)_{L^{\prime}}$, and introduce the sterile Majorana neutrinos $N=\left(N_{+}, N_{-}\right), s_{+}$, and $s_{-}$, whose left-handed components transform respectively as $(2,0)$, 
$(1,1)$, and $(1,-1)$ under $G_{L}$. A sterile scalar doublet $\phi$ transforming as $(2,1)$ is also added, whose VEV breaks $G_{L} \rightarrow U(1)_{e}$ where $L_{e}=2 T_{3}+L^{\prime}$. The renormalizable interactions predict three massless neutrinos $\nu_{e}^{\prime}, \nu_{\mu}$ and $\nu_{\tau}$, and two heavy Dirac neutrinos of mass $M$ and $\Lambda$, say. $\nu_{e}$ is mostly $\nu_{e}^{\prime}$, but mixes with $N_{+}$and $s_{+}$similarly to the previous model. There are three massless Goldstone boson states: a complex scalar $\varphi$ having charge $L_{e}(\varphi)=-2$ and a real scalar with $L_{e}=0$.

For $\beta \beta_{c M}$ the unbroken lepton number not only forbids contributions from graphs involving purely massless neutrinos, but it also causes the contribution from each heavyneutrino line to go like $1 / M^{2}$ rather than $1 / M$ when $M>100 \mathrm{MeV}$. The equivalent GR coupling of the Majoron in $\beta \beta_{c M}$ is roughly

$$
g_{\mathrm{eff}} \sim \sin ^{2} \theta \frac{M}{2 v} \frac{Q p_{F} M^{2}}{\left(p_{F}^{2}+M^{2}\right)^{2}} X
$$

if $M \sim \Lambda$ is the heavy neutrino mass and $v$ is the $G_{L}$-breaking scale. (cf. eq. (11), which due to the relation between $b_{i j}$ and the mass matrix does reproduce (14), despite appearances.) For $M \sim v \sim p_{F}, \theta \sim 0.1$ and $X \sim 1, g_{\text {eff }}$ is of order $\theta^{2} Q / p_{F} \sim 10^{-4}$ as desired.

To be more quantitative, we must evaluate the new nuclear matrix elements for $\beta \beta_{c M}$, which differ from the usual ones because the leptonic part of the amplitude is proportional to the Majoron and virtual neutrino momenta through the factor $[\not p, q]$. The $p_{0}$ piece $(\mathcal{V}$ of eq. (8)) vanishes in the integral $\int d p_{0}$, up to small $O(Q / p)$ corrections. To have a $0^{+} \rightarrow 0^{+}$nuclear transition, the $p_{i}$ piece $(\mathcal{U}$ in eq. (8)) must combine with odd-parity nuclear operators, which come from the recoil corrections to the nucleon currents and pwave Coulomb corrections to the electron wave functions. Although they are formally of higher order in $\alpha$ or $v / c$ of the nucleon than the usual Gamow-Teller and Fermi matrix elements, explicit calculations by nuclear theorists show that they need not all be small, as we will show [15].

For small mixing angles the matrix element becomes

$$
\left|\mathcal{A}\left(\beta \beta_{c M}\right)\right|^{2}=\frac{2}{\pi^{2}}\left(\theta g_{A}\right)^{4}\left(\frac{M}{v}\right)^{2} \sum_{i=1,2}\left|\mathcal{M}_{i}\right|^{2}
$$

in which the nuclear matrix elements are

$$
\left|\mathcal{M}_{1}\right|^{2}=\frac{7}{9}\left|\left\langle\left(A_{1}+i A_{2}\right) \tilde{h}\right\rangle\right|^{2} ; \quad\left|\mathcal{M}_{2}\right|^{2}=\frac{2}{9}\left|\left\langle A_{3} \tilde{h}\right\rangle\right|^{2}
$$


and $A_{i}$ are operators

$$
\begin{aligned}
A_{1} & =\hat{\mathbf{r}} \cdot\left[\left(C_{m} \sigma_{n}-C_{n} \sigma_{m}\right)+\left(g_{V} / g_{A}\right)^{2}\left(\mathbf{D}_{n}-\mathbf{D}_{m}\right)\right. \\
& \left.+i\left(g_{V} / g_{A}\right)\left(\mathbf{D}_{n} \times \sigma_{m}+\sigma_{n} \times \mathbf{D}_{m}\right)\right] ; \\
A_{2} & =\frac{\alpha Z r}{2 R}\left[\left(g_{V} / g_{A}\right)^{2}+\sigma_{m} \cdot \sigma_{n}-2 \sigma_{m} \cdot \hat{\mathbf{r}} \sigma_{n} \cdot \hat{\mathbf{r}}\right] ; \\
A_{3} & =\hat{\mathbf{r}} \cdot\left[\left(C_{m} \sigma_{n}+C_{n} \sigma_{m}\right)+\left(g_{V} / g_{A}\right)^{2}\left(\mathbf{D}_{n}+\mathbf{D}_{m}\right)\right] .
\end{aligned}
$$

We use the notation $\left\langle O_{n} O_{m}\right\rangle=\left\langle 0_{f}^{+}\left|\sum_{n, m} O_{n} O_{m} \tau_{n}^{+} \tau_{m}^{+}\right| 0_{i}^{+}\right\rangle$, where $\tau_{n}^{+}$is the isospin raising operator for the $n$th nucleon. Definitions of $C$ and $\mathbf{D}$ can be found in the reviews by Doi et al. [16] or Tomoda [17]. The neutrino potential $\tilde{h}$ depend on the internucleon separation vector $\mathbf{r}=\mathbf{r}_{n}-\mathbf{r}_{m}$ and the heavy neutrino mass $M$ :

$$
\tilde{h}=\frac{\partial}{\partial M^{2}} \frac{\partial}{\partial r}(h(0)-h(M)) ; \quad h(M)=\int \frac{d^{3} p}{2 \pi^{2}} e^{i \mathbf{p} \cdot \mathbf{r}} \frac{1}{\omega(\omega+\mu)},
$$

where $\omega=\left(p^{2}+M^{2}\right)^{1 / 2}$ and $\mu \sim 10 \mathrm{MeV}$ is the average nuclear excitation energy.

In Table 2 we show the value of the nuclear matrix elements, $\left(\sum_{i}\left|\mathcal{M}_{i}\right|^{2}\right)^{1 / 2}$, needed for charged Majoron emission to account for the anomalous events, assuming that $\theta^{2}(M / v)=$ $3 \times 10^{-2}$ (as would be the case for a neutrino with $17 \%$ mixing, the limit coming from flavor universality [18]). For comparison, we also show a similar matrix element that has been calculated by Muto et al. [19], namely

$$
\mathcal{M}_{R}=\left(g_{V} / g_{A}\right)\left\langle p_{F}^{2}\right\rangle^{-1}\left\langle\left(\mathbf{D}_{n} \times \sigma_{m}+\sigma_{n} \times \mathbf{D}_{m}\right) \cdot \hat{\mathbf{r}} \partial h / \partial r\right\rangle
$$

Except for a minor difference in the neutrino potential, the same matrix element is contained in the $A_{1}$ term of eq. (16). This is only meant to be indicative because there are further contributions to our $\sum_{i}\left|\mathcal{M}_{i}\right|^{2}$ which do not appear in the literature, and which are conceivably bigger than $\mathcal{M}_{R}$.

From table 2 we see that charged Majoron emission explains the data remarkably well, if the as-yet uncomputed matrix element for ${ }^{238} \mathrm{U}$ is similar to those of the other nuclei. In particular the $\beta \beta_{c M}$ rates for the geochemically observed decays of ${ }^{128} \mathrm{Te}$ and ${ }^{130} \mathrm{Te}$ dominate over the contribution of $\beta \beta_{2 \nu}$. As was described earlier, this is in better agreement with the data than is the result for $\beta \beta_{O M}$ models.

Because of the extra suppression by $\left(Q / p_{F}\right)^{2}$ in $\beta \beta_{c M}$, we needed a heavy neutrino $\Psi$ mixing with $\nu_{e}$ at the $\theta=10 \%$ level, with a mass near $100 \mathrm{MeV}$. But there are strong limits on $\theta$ for such a heavy neutrino. Of these, beam-dump experiments do not apply 
Table 2: phase space and matrix elements for charged Majoron emission in double beta decay. The phase space factors are defined analogously to those in table 1 . We give the values of the matrix elements which are needed to explain the data (assuming all events were $\beta \beta_{c M}$ for ${ }^{238} \mathrm{U}$ and Te) for $\theta^{2} M / v=3 \times 10^{-2}$, and values of some representative matrix elements that have been calculated; see eqs. (16) and (19), respectively.

\begin{tabular}{|c|c|c|c|c|}
\hline Element & $\Omega_{3} / m_{e}^{9}$ & $\Delta \Omega_{3} / m_{e}^{9}$ & $\left(\sum_{i}\left|\mathcal{M}_{i}\right|^{2}\right)^{1 / 2}$ needed & $\left|\mathcal{M}_{R}\right|$ (Muto et al. $)$ \\
\hline${ }^{76} \mathrm{Ge}$ & 4.7 & 0.43 & 1.1 & 1.1 \\
${ }^{82} \mathrm{Se}$ & 78 & 7.9 & 0.99 & 0.95 \\
${ }^{100} \mathrm{Mo}$ & 160 & 42 & 1.4 & 1.1 \\
${ }^{150} \mathrm{Nd}$ & 1570 & 320 & 1.6 & 1.3 \\
\hline${ }^{128} \mathrm{Te}$ & 0.13 & 0.13 & 0.32 & 0.92 \\
${ }^{130} \mathrm{Te}$ & 110 & 110 & 0.48 & 0.78 \\
${ }^{238} \mathrm{U}$ & 35 & 35 & 0.61 & $?$ \\
\hline
\end{tabular}

to our model because $\Psi$ decays invisibly into a light neutrino plus a Majoron. However, searches for peaks in the spectra of $\pi, K \rightarrow \nu e$ [20] rule out a long-lived heavy neutrino with such large mixing, unless $M>M_{K} \approx 500 \mathrm{MeV}$. For such large $M$ the $\beta \beta_{c M}$ rate becomes suppressed, unless the unknown nuclear matrix elements turn out to be considerably larger than those of ref. [19].

But there is a loophole in the peak search bounds: they do not apply if the $100 \mathrm{MeV}$ neutrino $\Psi$ is a broad resonance. A width of $10 \mathrm{MeV}$ is sufficient to hide the $\pi \rightarrow \nu \Psi$ peak beneath backgounds [21], and the $K$ decay searches do not extend below $140 \mathrm{MeV}$. Such large widths occur in our model if the neutrino-Majoron coupling is strong, $g \sim 4 \pi$, in which case we must repeat the $\beta \beta_{M}$ analysis with new form factors parameterizing the strongly-coupled neutrino sector. Then dimensional analysis again implies that the rate is maximal if the scale for new neutrino physics is of order $100 \mathrm{MeV}$. It would be fascinating if double beta decay experiments gave the first hint of a strongly-coupled neutrino sector!

We note that $v \sim M \sim$ (few hundred $\mathrm{MeV}$ ) is also the scale at which the global symmetry $G_{L}$ must break. While this is still annoyingly small compared to the weak scale, it is a significant improvement on the much smaller $10 \mathrm{keV}$ scales needed in ordinary Majoron models.

A final challenge confronting any model is the strong limit, $N_{\nu}<3.3$, 222] on the number of neutrino species at nucleosynthesis. The large coupling implied by the $\beta \beta$ anomalies means that Majorons had an undiluted thermal energy density at MeV temperatures, a 
potential disaster, since each Majoron counts as $4 / 7$ of a neutrino. The three Majoron states of the $\beta \beta_{c M}$ models therefore preclude more than two light neutrinos at nucleosynthesis, which could happen if $\nu_{\tau}$ (or $\nu_{\mu}$ ) decays before $0.8 \mathrm{MeV}$. One possibility is that $m_{\nu_{\tau}} \sim 25 \mathrm{MeV}$ (large enough to allow it to decouple before nucleosynthesis but still below the laboratory mass bound), with a lifetime $\tau<10^{3}$ sec [23]. But even if $\nu_{\tau}$ is light, it can effectively contribute as negative number of neutrino species if it decays into $\nu_{e} \varphi$ with lifetime $6 \times 10^{-4} \sec <\tau<2 \times 10^{-2} \sec [24]$, an even better prospect for our models. The first scenario, for example, occurs in our ordinary Majoron model via $\nu_{\tau} \rightarrow \nu_{\mu}+\varphi$, mediated by a dimension-seven operator $\left(L_{\mu} H\right) P_{L}\left(L_{\tau} H\right) \varphi^{*} \varphi$. Similarly, we can use dimension-five and dimension-seven interactions to give majorana masses and lifetimes to $\nu_{\mu}$ and $\nu_{\tau}$ in the charged Majoron model.

In summary, we have presented two classes of models for Majoron emission in double beta decay which may be able to explain excess events near the endpoints of several elements without seriously spoiling the agreement of geochemical $\beta \beta$ observations with theory. We propose a new class of models involving a 'charged' Majoron that carries an unbroken lepton number, which predict a softer sum energy spectrum than that of ordinary Majorons, and thus make them experimentally distinguishable from the latter. Charged-Majoron models are highly constrained, and require a larger, $100 \mathrm{MeV}$ scale of symmetry breaking than the unnaturally small $10 \mathrm{keV}$ scale of ordinary Majorons - a $10^{4}$ fold improvement. The detailed predictions for these models depend in part on the size of certain nuclear matrix elements (eq. (16)) which differ from those appearing in the usual amplitudes for $\beta \beta_{2 \nu}, \beta \beta_{0 \nu}$, or $\beta \beta_{O M}$. All our models point toward a sterile neutrino with a mass of a few hundred $\mathrm{MeV}$, a large $(\sim 0.1)$ angle for mixing with $\nu_{e}$, and possibly a large $(\sim 10 \mathrm{MeV})$ width. Nucleosynthesis requirements suggest that the neutrino which is predominantly $\nu_{\tau}$ should either have a mass that is not much below the laboratory lower limit, or else a lifetime $\lesssim 10^{-3}$ sec.

We warmly thank F. Avignone, M. Moe and A. Turkevich for information about their experiments, T. Kotani, R. Shrock and E. Takasugi for helpful correspondence, W. Haxton, M. Luty, N. de Takacsy and P. Vogel for helpful discussions and R. Fernholz for his contributions,including the figure. This work was supported in part by the Natural Sciences and Engineering Research Council of Canada and les Fonds F.C.A.R. du Québec. 


\section{References}

[1] Y. Chikashige, R.N. Mohapatra and R.D. Peccei, Phys. Lett. 99B (1981) 411.

[2] M. Doi, T. Kotani and E. Takasugi, Phys. Rev. D37 (1988) 2572.

[3] H.M. Georgi, S.L. Glashow and S. Nussinov, Nucl. Phys. B193 (1981) 297.

[4] F.T. Avignone III et al., in Neutrino Masses and Neutrino Astrophysics, proceedings of the IV Telemark Conference, Ashland, Wisconsin, 1987, edited by V. Barger, F. Halzen, M. Marshak and K. Olive (World Scientific, Sinagpore, 1987), p. 248.

[5] P. Fisher et al., Phys. Lett. B192 (1987) 460; D.O. Caldwell et al., Phys. Rev. Lett. 59 (1987) 1649.

[6] M. Moe, M. Nelson, M. Vient and S. Elliott, preprint UCI-NEUTRINO 92-1.

[7] F.T. Avignone, private communication

[8] G.B. Gelmini and M. Roncadelli, Phys. Lett. 99B (1981) 411.

[9] We have been informed that the discrepancy between the ${ }^{238} \mathrm{U}$ observations of Turkevich, Economou and Cowan, (Phys. Rev. Lett. 67 (1991) 3211) and the calculations of Staudt, Muto and Klapdor-Kleingrothaus (Europhys. Lett., 13 (1990) 31) have now been resolved.

[10] It has recently been claimed by Z.G. Berezhiani, A.Yu. Smirnov and J.W.F. Valle, (preprint FTUV/92-20, IFIC/92-21, LMU-05/92) that the required Majoron coupling, $g_{M} \sim 10^{-4}$, is sufficiently small to allow such a hierarchy below the weak scale. This argument overlooks the fact that in these models the effective Majoron coupling measured in $\beta \beta_{M}$ decay is given by $g_{M} \sim g \theta^{2}$, where the small mixing angle, $\theta$, is bounded by oscillation and neutrino decay experiments to be very small. Since the coupling that controls the hierarchy problem is $g$, and not $g_{M}$, it is typically not small: $g \sim O(1)$.

[11] F.T. Avignone III et al., Phys. Lett. B256 (1991) 559.

[12] This result of Bernatowicz and Holenberg, together with its application to a $\beta \beta_{O M}$ description of the excess events may be found in the talk by W. Haxton, at Neutrino 92, Granada, Spain

[13] R.E. Shrock, Phys. Rev. D24 (1981) 1232; T. Yamazaki et al., Proceedings of the XIth International Conference on Neutrino Physics and Astrophysics, eds. K. Kleinknecht and E.A. Paschos (World Scientific, Singapore, 1984), p. 183.

[14] D.I. Britton et al., Phys. Rev. Lett. 68 (1992) 3000.

[15] The differences between charged and ordinary Majorons can be seen in ref. [2], in what they call the "correction term;" in contrast to their general analysis, our model has part of this correction term being the leading contribution. Our results cannot simply be extracted from ref. [2] because we differ on the form of the neutrino potential, and in having the matrix element $\mathcal{M}_{2}$.

[16] M. Doi, T. Kotani and E. Takasugi, Prog. Theo Phys. Suppl. 83 (1985) 1.

[17] T. Tomoda, Rept. Prog. Phys. 54 (1991) 53. 
[18] M. Gronau, C.N. Leung and J.L. Rosner, Phys. Rev. D 29, (1984) 2539.

[19] K. Muto, E. Bender and H.V. Klapdor, Z. Phys. A 334 (1989) 187, as tabulated in 177.

[20] R.E. Shrock, Phys. Rev. D24 (1981) 1232; T. Yamazaki et al., Proceedings of the XIth International Conference on Neutrino Physics and Astrophysics, eds. K. Kleinknecht and E.A. Paschos (World Scientific, Singapore, 1984), p. 183.

[21] We thank D. Britton for helpful conversations on this point.

[22] T.P. Walker, G. Steigman, D.N. Schramm, K.A. Olive and H.S. Kang, Ap. J. 376 (1991) 51 and references therein.

[23] E.W. Kolb, M.S. Turner, A. Chakravorty and D.N. Schramm, Phys. Rev. Lett. 67 (1991) 533.

[24] K. Enqvist, K. Kainulainen and M. Thomson, Phys. Rev. Lett. 68 (1992) 744. 